Research Article

\title{
Minimum Detour Index of Tricyclic Graphs
}

\author{
Wei Fang $\mathbb{D},{ }^{1,2}$ Zheng-Qun Cai $\mathbb{D}^{3},{ }^{3}$ and Xiao-Xin $\operatorname{Li} \mathbb{C}^{4}$ \\ ${ }^{1}$ School of Mathematical Sciences, Anhui University, Hefei 230601, China \\ ${ }^{2}$ College of Information \& Network Engineering, Anhui Science and Technology University, Fengyang 233100, China \\ ${ }^{3}$ School of Foreign Studies, Anhui Jianzhu University, Hefei 230601, China \\ ${ }^{4}$ School of Big Data and Artificial Intelligence, Chizhou University, Chizhou 247000, China \\ Correspondence should be addressed to Zheng-Qun Cai; caizhengqun1983@163.com
}

Received 7 July 2019; Accepted 5 September 2019; Published 13 October 2019

Guest Editor: Shaohui Wang

Copyright (c) 2019 Wei Fang et al. This is an open access article distributed under the Creative Commons Attribution License, which permits unrestricted use, distribution, and reproduction in any medium, provided the original work is properly cited.

The detour index of a connected graph is defined as the sum of the detour distances (lengths of longest paths) between unordered pairs of vertices of the graph. The detour index is used in various quantitative structure-property relationship and quantitative structure-activity relationship studies. In this paper, we characterize the minimum detour index among all tricyclic graphs, which attain the bounds.

\section{Introduction}

Let $G$ be a simple and connected graph with $|V(G)|=n$ and $|E(G)|=m$ and $N_{G}(u)$ be the neighbor vertex set of vertex $u$, then $d_{G}(u)=\left|N_{G}(u)\right|$ is called the degree of $u$. If $m=n-1+c$, then $G$ is called a $c$-cyclic graph. If $c=0,1,2$, and 3 , then $G$ is a tree, unicyclic graph, bicyclic graph, and tricyclic graph, respectively. Denote by $\mathscr{T}_{n}$ the set of all tricyclic graphs of order $n$.

Let $\widehat{\mathscr{T}}=\left\{\widehat{\mathscr{T}}^{i} \mid 1 \leq i \leq 15\right\}$, where graphs $\hat{\mathscr{T}}^{i}$ for $i=1,2, \ldots, 15$ are defined in Figure 1 . By [1,2], we know that for any $\mathscr{T}^{i} \in \mathscr{T}_{n}, \quad \mathscr{T}^{i}$ can be obtained from $\hat{\mathscr{T}}^{i}(1 \leq i \leq 15)$ by attaching trees to some of its vertices. We call $\hat{\mathscr{T}}^{i}$ the base of $\mathscr{T}^{i}$.

A block of the graph $G$ is a maximal 2-connected subgraph of $G$. A cactus is a connected graph in which no edge lies in more than one cycle, such that each block of a cactus is either an edge or a cycle. A vertex shared by two or more cycles is called a cut vertex. In this paper, denote $\mathscr{C}_{n}^{l}$ be the set of all cacti of order $n$ and $l$ cycles, where $l \geq 1$. The length of the cycles may be different and the length of each cycle is at least 3.

The concept of "topological index" was first proposed by Haruo Hosoya for characterizing the topological nature of a graph. Such graph invariants are usually related to the distance function $d(-,-)$.
The detour distance $[3,4]$ (also known under the name elongation) between vertices $u$ and $v$ in $G$ is the length of a longest path between them, denoted by $l(u, v \mid G)$. Note that $l(u, u \mid G)=0$ for any $u \in V(G)$; see [5] for a discussion. The detour index of the graph $G$ is defined as [4-9]

$$
\omega(G)=\frac{1}{2} \sum_{u, v \in V(G)} l(u, v \mid G) .
$$

For a connected graph $G$ with $u \in V(G)$, let $L(u \mid G)=$ $\sum_{v \in V(G)} l(u, v \mid G)$, then

$$
\omega(G)=\frac{1}{2} \sum_{u \in V(G)} L(u \mid G) .
$$

If we use the notion of the detour matrix [4], which is an $n \times n$ matrix whose $(i, j)$-element is $l\left(v_{i}, v_{j} \mid G\right)$ with $V(G)=\left\{v_{1}, v_{2}, \ldots, v_{n}\right\}$, then the detour index is equal to the half-sum of the (off diagonal) elements of the detour matrix. The detour index has been applied to chemistry, especially in quantitative structure-activity relationship (QSAR) studies; see $[7,10]$ for more details. A new branch cheminformatics is a combination of mathematics and chemistry. This branch studies QSAR/QSPR study, physicochemical properties and topological indices such as Zagreb Indices [11], Kirchhoff index [12], Hosoya index [13] and so on to predict 


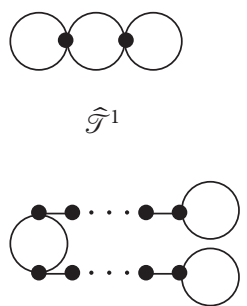

$\widehat{\mathscr{T}}^{5}$

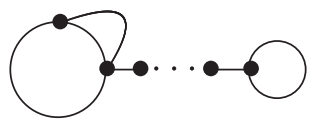

$\widehat{\mathscr{T}}^{10}$
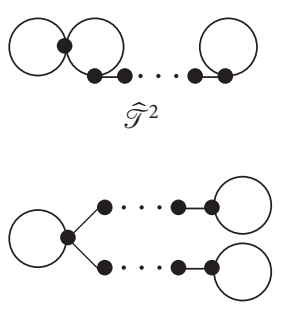

$\hat{\mathscr{T}}^{6}$

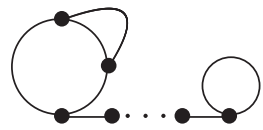

$\widehat{\mathscr{T}}^{11}$

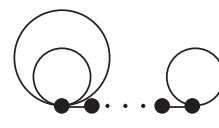

$\widehat{\mathscr{T}}^{3}$

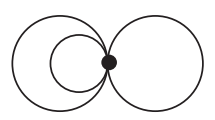

$\hat{\mathscr{T}}^{7}$

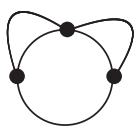

$\widehat{\mathscr{T}}^{12}$
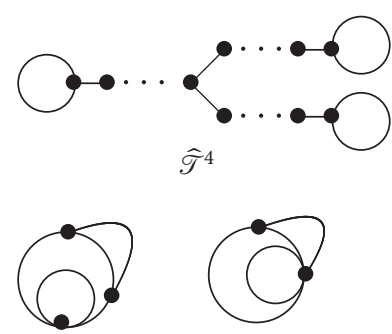

$\widehat{\mathscr{T}}^{8}$

$\widehat{\mathscr{T}}^{9}$

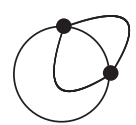

$\widehat{\mathscr{T}}^{13}$

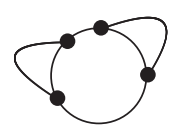

$\widehat{\mathscr{T}}^{14}$

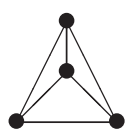

$\widehat{\mathscr{T}}^{15}$

FIgURE 1: The fifteen types of bases for tricyclic graphs.

physicochemical properties and biological activities of the chemical compounds theoretically.

In this paper, we consider the minimum detour index among all tricyclic graphs.

\section{Preliminaries}

In this section, we will introduce some useful lemmas and graph transformations.

2.1. Edge-Lifting Transformation $[14,15]$. Let $G_{1}$ and $G_{2}$ be two graphs with $n_{1} \geq 2$ and $n_{2} \geq 2$ vertices, respectively. If $G$ is the graph obtained from $G_{1}$ and $G_{2}$ by adding an edge between a vertex $u_{0}$ of $G_{1}$ and a vertex $v_{0}$ of $G_{2}, G^{\prime}$ is the graph obtained by identifying $u_{0}$ of $G_{1}$ to $v_{0}$ of $G_{2}$ and adding a pendant edge to $u_{0}\left(v_{0}\right)$, then $G^{\prime}$ is called the edge-lifting transformation of $G$ (see Figure 2).

Lemma 1 (see [16]). Let $G$ be defined as in Figure 2, and $G^{\prime}$ is obtained from $G$ by the edge-lifting transformation (see Figure 2). Then, $\omega(G)>\omega\left(G^{\prime}\right)$.

Denote $\quad \mathscr{T}_{n}^{(1)}=\left\{\mathscr{T}^{1}, \mathscr{T}^{7}, \mathscr{T}^{8}, \mathscr{T}^{9}, \mathscr{T}^{12}, \mathscr{T}^{13}, \mathscr{T}^{14}, \mathscr{T}^{15}\right\}$ (see Figure 1).

By Lemma 1, we can verify that if $\mathscr{T} \in \mathscr{T}_{n}$ attains the minimum detour index of all graphs in $\mathscr{T}_{n}$, then the following two conditions hold:

(i) The base $\hat{\mathscr{T}}$ of $\mathscr{T}$ is one of $\hat{\mathscr{T}}_{n}^{(1)}$

(ii) The graph $\mathscr{T}$ is obtained from $\widehat{\mathscr{T}}$ by attaching some pendant edges

Remark 1. In order to determine the tricyclic graphs which attain the minimum detour index of all graphs in $\mathscr{T}_{n}$, we just need to discuss the tricyclic graphs in $\mathscr{T}$, where $\mathscr{\mathscr { T }}_{n} \in \mathscr{T}_{n}^{(1)}$.

2.2. Cycle-Edge Transformation. Let $\mathscr{C} \in \mathscr{C}_{n}^{l}$ be a cactus as shown in Figure 3, where $C_{r}=v_{1} v_{2} \ldots v_{r} v_{1}$ is the biggest cycle of $\mathscr{C}, r \geq 4$. Denote the vertex set $W_{v_{i}}=$
$N_{G_{i}}\left(v_{i}\right)=N_{G}\left(v_{i}\right) \cap V\left(G_{i}\right), 1 \leq i \leq r . \mathscr{C}^{\prime}$ is the graph obtained from $\mathscr{C}$ by deleting the edges $v_{2} v_{3}$ and $v_{2}$ to $W_{v_{2}}$, meanwhile adding the edges $v_{1} v_{3}$ and $v_{1}$ to $W_{v_{2}}$.

We say that $\mathscr{C}^{\prime}$ is obtained from $\mathscr{C}$ by the cycle-edge transformation (see Figure 3).

Lemma 2 (see [16]). Let $\mathscr{C} \in \mathscr{C}_{n}^{l}$ be a cactus as shown in Figure 3 with $r \geq 4$, and $\mathscr{C}^{\prime}$ be the cycle-edge transformation of $\mathscr{C}$ (see Figure 3). Then, $\omega(\mathscr{C})>\omega\left(\mathscr{C}^{\prime}\right)$.

2.3. Cycle-Lifting Transformation. Let $\mathscr{C}_{1} \in \mathscr{C}_{n}^{l}$ be a cactus as shown in Figure 4 . Denote $W_{v_{i}}=N_{G_{i}}\left(v_{i}\right)=N_{G}\left(v_{i}\right) \cap V\left(G_{i}\right)$ for $1 \leq i \leq 3$. Let $\mathscr{C}_{1}^{\prime}$ be the graph obtained from $\mathscr{C}_{1}$ by deleting the edges $v_{2} v_{x}$ for $v_{x} \in W_{v_{2}}$ and adding the edges $v_{1} v_{x}$ for $v_{x} \in W_{v_{2}}$.

We say that $\mathscr{C}_{1}^{\prime}$ is the cycle-lifting transformation of $\mathscr{C}_{1}$ (see Figure 4).

Lemma 3 (see [16]). Let $\mathscr{C}_{1}^{\prime}$ be the cycle-lifting transformation of $\mathscr{C}_{1}$ (see Figure 4$)$. Then, $\omega\left(\mathscr{C}_{1}\right)>\omega\left(\mathscr{C}_{1}^{\prime}\right)$.

2.4. Operation I. We define Operation I as follows. Let $G$ and $G^{\prime}$ be a simple and connected graph as shown in Figure 5. $v_{1} v_{2} \ldots v_{p}$ be the path in a cycle. Denote $W_{v_{i}}=$ $\left\{w \mid w v_{i} \in E(G)\right.$ and $\left.d(w)=1,1 \leq i \leq p, p \geq 3\right\}$, and $G^{\prime}$ be the graph obtained from $G$ by deleting the edges $v_{2} v_{3}, v_{2} w$ for $w \in W_{v_{2}}$ and adding the edges $v_{1} v_{3}, v_{1} w$ for $w \in W_{v_{2}}$ (see Figure 5).

Lemma 4. Let $G$ and $G^{\prime}$ be the graph shown in Figure 5. Then, $\omega(G)>\omega\left(G^{\prime}\right)$.

Proof. Let $V(G)=V\left(G^{\prime}\right)=\left\{v_{1}, v_{2}, v_{3}, \ldots, v_{n}\right\}$, and $W_{v_{i}}=$ $\left\{w \mid w v_{i} \in E(G)\right.$ and $\left.d(w)=1,1 \leq i \leq p\right\}$. For the vertices $v_{i}, v_{j} \in V\left(G-v_{2}\right)$, obviously 


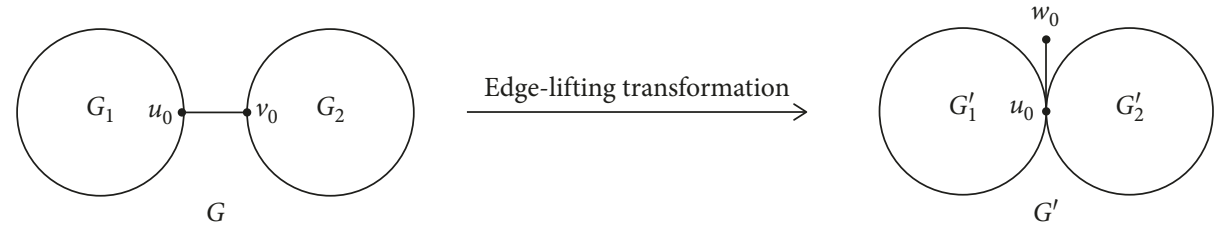

FIgure 2: The edge-lifting transformation.

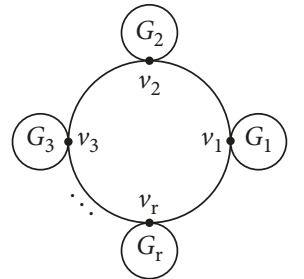

$\mathscr{C}$
Cycle-edge transformation

Cycle-lifting transformation

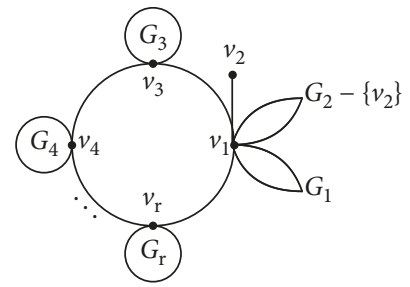

$\mathscr{C}^{\prime}$

FIGURE 3: The cycle-edge transformation.
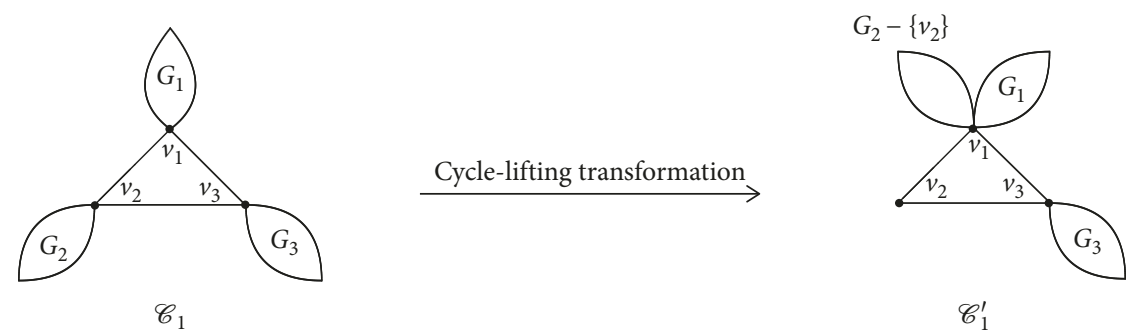

FIgURE 4: The cycle-lifting transformation.

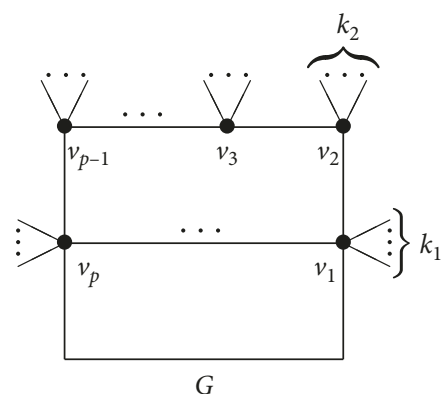

$\stackrel{\text { Operation I }}{\longrightarrow}$

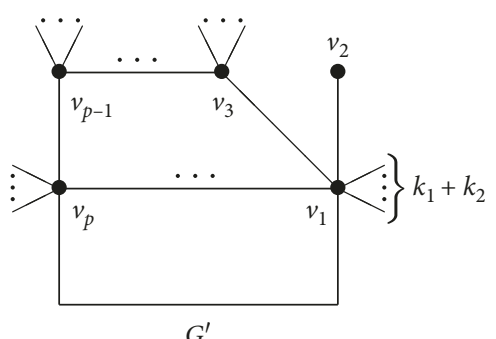

FIgURE 5: Operation I on graph $G$.

$$
\begin{aligned}
& l\left(v_{i}, v_{j} \mid G\right) \geq l\left(v_{i}, v_{j} \mid G^{\prime}\right), \\
& l\left(v_{1}, v_{2} \mid G\right)>l\left(v_{1}, v_{2} \mid G^{\prime}\right)=1 .
\end{aligned}
$$

Let $P_{i}$ be the set of the longest path between $v_{2}$ and $v_{i}$ in $G$ and $Q_{i}$ be the set of the longest path between $v_{1}$ and $v_{i}$ in $G$, where $3 \leq i \leq n$.

Case 1. $v_{1} v_{2} \in E\left(P_{i}\right)$ and $v_{2} v_{3} \in E\left(P_{i}\right)$, where $3 \leq i \leq n$.

Obviously, for $3 \leq i \leq n, l\left(v_{2}, v_{i} \mid G\right)=l\left(v_{2}, v_{i} \mid G^{\prime}\right)-1$. On the other hand, if $v_{1} v_{2} \in E\left(P_{i}\right)$ and $v_{2} v_{3} \in E\left(P_{i}\right)$, then $v_{1} v_{2}, v_{2} v_{3} \in \mathscr{L}_{i}$, where $\mathscr{L}_{i}$ be the any one longest path between $v_{1}$ and $v_{i}$ in $G$. Therefore, $l\left(v_{1}, v_{i} \mid G\right)=l\left(v_{1}, v_{i} \mid G^{\prime}\right)+1$, and

$$
\begin{aligned}
& l\left(v_{2}, v_{i} \mid G\right)+l\left(v_{1}, v_{i} \mid G\right) \\
& \quad=l\left(v_{2}, v_{i} \mid G^{\prime}\right)+l\left(v_{1}, v_{i} \mid G^{\prime}\right), \quad 3 \leq i \leq n .
\end{aligned}
$$

Case 2. $v_{1} v_{2} \notin E\left(P_{i}\right)$ and $v_{2} v_{3} \in E\left(P_{i}\right)$. Obviously, for $3 \leq i \leq n$, we have

$$
\begin{aligned}
l\left(v_{2}, v_{i} \mid G\right) & =l\left(v_{3}, v_{i} \mid G\right)+1 \\
& >l\left(v_{1}, v_{i} \mid G\right)+1 \\
& \geq l\left(v_{1}, v_{i} \mid G^{\prime}\right)+1 \\
& =l\left(v_{2}, v_{i} \mid G^{\prime}\right) .
\end{aligned}
$$

Case 3. $v_{1} v_{2} \in E\left(P_{i}\right)$ and $v_{2} v_{3} \notin E\left(P_{i}\right)$. Obviously, for $3 \leq i \leq n$, we have

$$
l\left(v_{2}, v_{i} \mid G\right)=l\left(v_{2}, v_{i} \mid G^{\prime}\right) .
$$


Case 4. $v_{1} v_{2} \notin E\left(P_{i}\right)$ and $v_{2} v_{3} \notin E\left(P_{i}\right)$.

Obviously, $v_{i} \in W_{v_{2}} \subset V(G)$, and

$$
\begin{aligned}
& l\left(v_{2}, v_{i} \mid G\right)=l\left(v_{1}, v_{i} \mid G^{\prime}\right)=1, \\
& l\left(v_{1}, v_{i} \mid G\right)>l\left(v_{2}, v_{i} \mid G^{\prime}\right)=2 .
\end{aligned}
$$

By (5)-(9), we have

$$
\begin{aligned}
& l\left(v_{2}, v_{i} \mid G\right)+l\left(v_{1}, v_{i} \mid G\right) \\
& \quad \geq l\left(v_{2}, v_{i} \mid G^{\prime}\right)+l\left(v_{1}, v_{i} \mid G^{\prime}\right), \quad 3 \leq i \leq n .
\end{aligned}
$$

By (3), (4), and (10), we have $\omega(G)>\omega\left(G^{\prime}\right)$.

2.5. Operation II. We define Operation II as follows. Let $G$ and $G^{\prime}$ be a simple and connected graph as shown in Figure 6. Denote $v_{1} v_{2} v_{3} v_{1}$ be a cycle with length 3, $W_{v_{i}}=\left\{w \mid w v_{i} \in E(G)\right.$ and $\left.d(w)=1, i=1,2\right\}$, and $G^{\prime}$ be the graph obtained from $G$ by deleting the edges $v_{2} w$ for $w \in W_{v_{2}}$ and adding the edges $v_{1} w$ for $w \in W_{v_{2}}$ (see Figure 6).

Lemma 5. Let $G$ and $G^{\prime}$ be the graph shown in Figure 6. Then, $\omega(G)>\omega\left(G^{\prime}\right)$, and the equality holds if and only if $G \cong G^{\prime}$.

Proof. Let $V(G)=V\left(G^{\prime}\right)=\left\{v_{1}, v_{2}, v_{3}, \ldots, v_{n}\right\}$. For the vertices $v_{i}, v_{j} \in V(G)-W_{v_{2}}, v_{x}, v_{y} \in W_{v_{2}}$, we have

$$
\begin{aligned}
& l\left(v_{i}, v_{j} \mid G\right)=l\left(v_{i}, v_{j} \mid G^{\prime}\right), \\
& l\left(v_{x}, v_{y} \mid G\right)=l\left(v_{x}, v_{y} \mid G^{\prime}\right)=2 .
\end{aligned}
$$
have

For the vertices $v_{i} \in V(G)-W_{v_{2}}-\left\{v_{1}, v_{2}\right\}, w \in W_{v_{2}}$, we

$$
\begin{aligned}
l(w & \left., v_{i} \mid G\right) \\
& =\max \left\{3+l\left(v_{1}, v_{i} \mid G\right), 3+l\left(v_{3}, v_{i} \mid G\right)\right\} \\
& \geq \max \left\{1+l\left(v_{1}, v_{i} \mid G\right), 3+l\left(v_{3}, v_{i} \mid G\right)\right\} \\
& =\max \left\{1+l\left(v_{1}, v_{i} \mid G^{\prime}\right), 3+l\left(v_{3}, v_{i} \mid G^{\prime}\right)\right\} \\
& =l\left(w, v_{i} \mid G^{\prime}\right),
\end{aligned}
$$

especially, if $G \not G^{\prime}$, then

$$
\begin{aligned}
l\left(w, v_{3} \mid G\right) & >l\left(w, v_{3} \mid G^{\prime}\right), \\
l\left(w, v_{1} \mid G\right) & =l\left(v_{1}, v_{2} \mid G\right)+1 \\
& =l\left(v_{1}, v_{2} \mid G^{\prime}\right)+1 \\
& =l\left(w, v_{2} \mid G^{\prime}\right), \\
l\left(w, v_{2} \mid G\right) & =l\left(w, v_{1} \mid G^{\prime}\right)=1 .
\end{aligned}
$$

By (11)-(16), we have $\omega(G) \geq \omega\left(G^{\prime}\right)$, and the equality holds if and only if $G \cong G^{\prime}$.

Denote $\quad \mathscr{T}_{n}^{(2)}=\left\{\mathscr{T}^{16}, \mathscr{T}^{17}, \mathscr{T}^{18}, \mathscr{T}^{19}, \mathscr{T}^{20}, \mathscr{T}^{21}\right\} ; \quad$ see Figure 7.

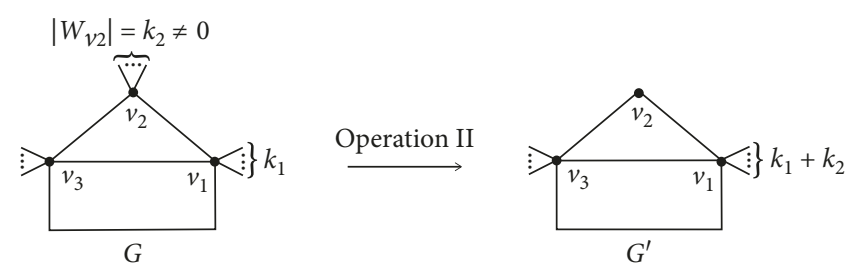

Figure 6: Operation II on graph G.

By Lemma 2-5, we can verify that if $\mathscr{T} \in \mathscr{T}_{n}$ attains the minimum detour index of all graphs in $\mathscr{T}_{n}$, then $\mathscr{T} \in \mathscr{T}_{n}^{(2)}$.

Remark 2. In order to determine the tricyclic graphs which attain the minimum detour index of all graphs in $\mathscr{T}_{n}$, we just need to discuss the tricyclic graphs in $\mathscr{T}$, where $\widehat{\mathscr{T}} \in \mathscr{T}_{n}^{(2)}=\left\{\mathscr{T}^{16}, \mathscr{T}^{17}, \mathscr{T}^{18}, \mathscr{T}^{19}, \mathscr{T}^{20}, \mathscr{T}^{21}\right\}$; see Figure 7 .

2.6. Operation III. We define Operation III as follows. Let $G \in \mathscr{T}^{17} \cup \mathscr{T}^{18} \cup \mathscr{T}^{20} \cup \mathscr{T}^{21}$ as shown in Figure 7. Denote $W_{v_{i}}=\left\{w \mid w v_{i} \in E(G)\right.$ and $\left.d(w)=1\right\}$ and $G^{\prime}$ be the graph obtained from $G$ by deleting the edges $v_{3} w$ for $w \in W_{v_{3}}$ and adding the edges $v_{1} w$ for $w \in W_{v_{3}}$ (see Figures 8-11).

Lemma 6. Let $G_{i}$ and $G_{i}^{\prime}(1 \leq i \leq 4)$ be the graph in Figures 8-11. Then, $\omega(G) \geq \omega\left(G^{\prime}\right)$, and the equality holds if and only if $G \cong G^{\prime}$.

Proof. Let $V\left(G_{1}\right)=V\left(G_{1}^{\prime}\right)=\left\{v_{1}, v_{2}, v_{3}, \ldots, v_{n}\right\}$. For the vertices $v_{i}, v_{j} \in V(G)-W_{v_{3}}-\left\{v_{1}, v_{3}\right\} ; v_{x}, v_{y} \in W_{v_{3}}$, we have

$$
\begin{aligned}
l\left(v_{i}, v_{j} \mid G_{1}\right) & =l\left(v_{i}, v_{j} \mid G_{1}^{\prime}\right), \\
l\left(v_{x}, v_{y} \mid G_{1}\right) & =l\left(v_{x}, v_{y} \mid G_{1}^{\prime}\right)=2, \\
l\left(v_{1}, v_{i} \mid G_{1}\right) & =l\left(v_{1}, v_{i} \mid G_{1}^{\prime}\right), \\
l\left(v_{3}, v_{x} \mid G_{1}\right) & =l\left(v_{1}, v_{x} \mid G_{1}^{\prime}\right), \\
l\left(v_{3}, v_{i} \mid G_{1}\right) & \geq l\left(v_{1}, v_{i} \mid G_{1}^{\prime}\right), \\
l\left(v_{x}, v_{i} \mid G_{1}\right) & =l\left(v_{3}, v_{i} \mid G_{1}\right)+1 \\
& =l\left(v_{3}, v_{i} \mid G_{1}^{\prime}\right)+1 \\
& \geq l\left(v_{1}, v_{i} \mid G_{1}^{\prime}\right)+1 \\
& =l\left(v_{x}, v_{i} \mid G_{1}^{\prime}\right), \\
l\left(v_{x}, v_{5} \mid G_{1}\right) & =5>3=l\left(v_{x}, v_{5} \mid G_{1}^{\prime}\right) .
\end{aligned}
$$

By (17)-(23), we have $\omega\left(G_{1}\right)>\omega\left(G_{1}^{\prime}\right)$.

Similarly, we have $\omega\left(G_{i}\right) \geq \omega\left(G_{i}^{\prime}\right)$, and the equality holds if and only if $G_{i} \cong G_{i}^{\prime}(i=2,3,4)$.

2.7. Operation IV. We define Operation IV as follows. Let $G \in \mathscr{T}^{19}$ as shown in Figure 12. Denote $W_{v_{i}}=\left\{w \mid w v_{i}\right.$ $\in E(G)$ and $d(w)=1\}$, and $G^{\prime}$ be the graph obtained from $G$ 


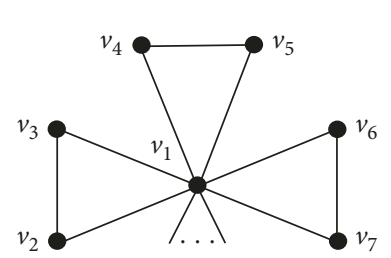

$\mathscr{T}^{16}$

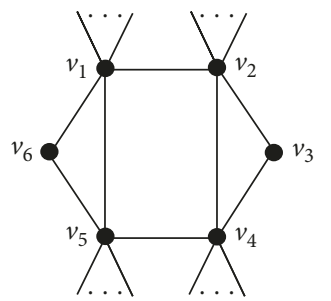

$\mathscr{T}^{19}$

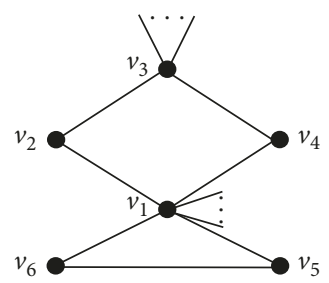

$\mathscr{T}^{17}$

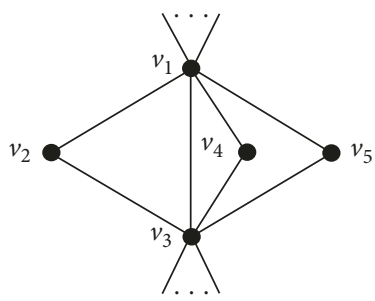

$\mathscr{T}^{20}$
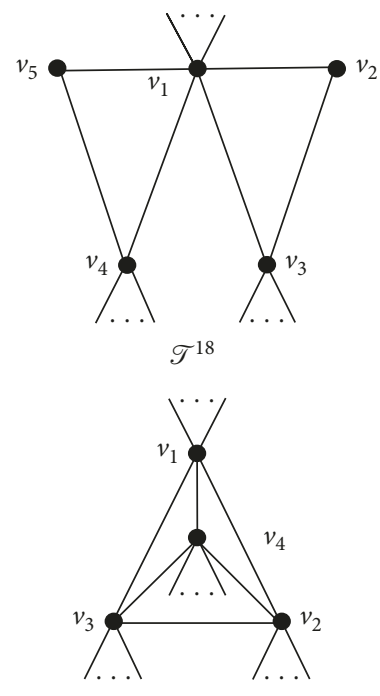

$\mathscr{T}^{21}$

FIgURE 7: Graph $\mathscr{T}_{n}^{(2)}$.

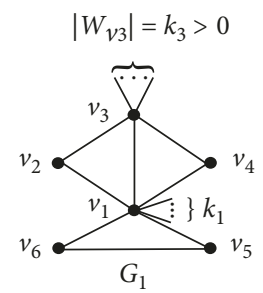

Operation III

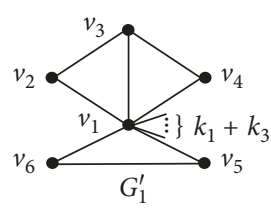

Figure 8: Operation III on graph $G_{1} \in \mathscr{T}^{17}$.

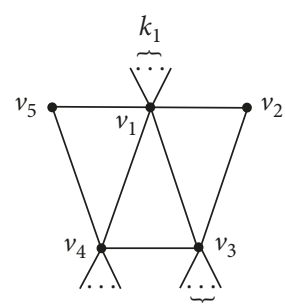

$\left|W_{v 3}\right|=k_{3}>0$

$G_{2}$
Operation III

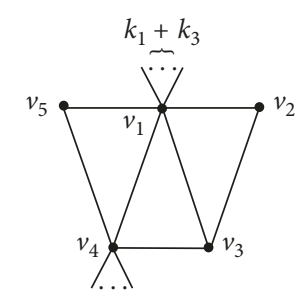

$G_{2}^{\prime}$

Figure 9: Operation III on graph $G_{2} \in \mathscr{T}^{18}$.

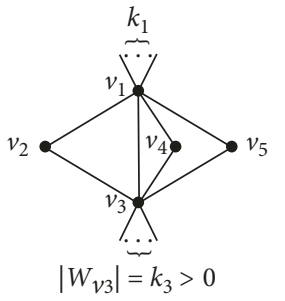

$G_{3}$

\section{$\underset{\mathrm{Operation} \text { III }}{\longrightarrow}$}

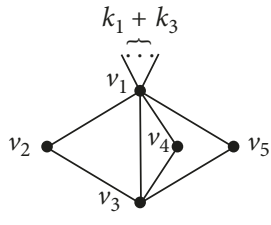

FIgURE 10: Operation III on graph $G_{3} \in \mathscr{T}^{20}$. 


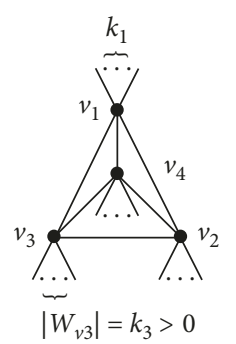

$G_{4}$

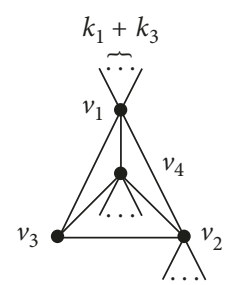

$G_{4}^{\prime}$

FIGURE 11: Operation III on graph $G_{4} \in \mathscr{T}^{21}$.

by deleting the edges $v_{i} w$ for $w \in W_{v_{i}}, i=2,4,5$ and adding the edges $v_{1} w$ for $w \in W_{v_{i}}, i=2,4,5$ (see Figure 12).

Lemma 7. Let $G$ and $G^{\prime}$ be the graph shown in Figure 12. Then, $\omega(G) \geq \omega\left(G^{\prime}\right)$ with equality holding if and only if $G \cong G^{\prime}$.

Proof. Let $V_{1}=\left\{v_{i} \mid 1 \leq i \leq 6\right\}, V_{2}=V(G)-V_{1}$, we have

$$
\begin{aligned}
\omega(G)= & \sum_{v_{x}, v_{y} \in V_{1}} l\left(v_{x}, v_{y} \mid G\right) \\
& +\sum_{v_{x} \in V_{1}, v_{y} \in V_{2}} l\left(v_{x}, v_{y} \mid G\right) \\
& +\sum_{v_{x}, v_{y} \in V_{2}} l\left(v_{x}, v_{y} \mid G\right), \\
\omega\left(G^{\prime}\right)= & \sum_{v_{x}, v_{y} \in V_{1}} l\left(v_{x}, v_{y} \mid G^{\prime}\right) \\
& +\sum_{v_{x} \in V_{1}, v_{y} \in V_{2}} l\left(v_{x}, v_{y} \mid G^{\prime}\right) \\
& +\sum_{v_{x}, v_{y} \in V_{2}} l\left(v_{x}, v_{y} \mid G^{\prime}\right) .
\end{aligned}
$$

Obviously,

$$
\begin{aligned}
& \sum_{v_{x}, v_{y} \in V_{1}} l\left(v_{x}, v_{y} \mid G\right)=\sum_{v_{x}, v_{y} \in V_{1}} l\left(v_{x}, v_{y} \mid G^{\prime}\right), \\
& \sum_{v_{x} \in V_{1}, v_{y} \in V_{2}} l\left(v_{x}, v_{y} \mid G\right)=+\sum_{v_{x} \in V_{1}, v_{y} \in V_{2}} l\left(v_{x}, v_{y} \mid G^{\prime}\right), \\
& l\left(v_{x}, v_{y} \mid G\right)=l\left(v_{x}, v_{y} \mid G^{\prime}\right)=2, \quad v_{x}, v_{y} \in W_{v_{i}}, \\
& l\left(v_{x}, v_{y} \mid G\right)>2=l\left(v_{x}, v_{y} \mid G^{\prime}\right), \\
& v_{x} \in W_{v_{i}}, v_{y} \in W_{v_{j}}, i \neq j .
\end{aligned}
$$

Therefore,

$$
\begin{aligned}
& \omega(G)-\omega\left(G^{\prime}\right) \\
& =\sum_{v_{x}, v_{y} \in V_{2}} l\left(v_{x}, v_{y} \mid G\right)-\sum_{v_{x}, v_{y} \in V_{2}} l\left(v_{x}, v_{y} \mid G^{\prime}\right) \\
& \quad \geq 0,
\end{aligned}
$$

and the equality holds if and only if $G \cong G^{\prime}$.

Denote $\quad \mathscr{T}_{n}^{(3)}=\left\{\mathscr{T}^{16} \cup \mathscr{T}^{22} \cup \mathscr{T}^{23} \cup \mathscr{T}^{24} \cup \mathscr{T}^{25} \cup \mathscr{T}^{26}\right\}$ (see Figure 13).

By Lemma 6-7, we can verify that if $\mathscr{T} \in \mathscr{T}_{n}$ attains the minimum detour index of all graphs in $\mathscr{T}_{n}$, then $\widehat{\mathscr{T}}_{\in} \in \mathscr{T}_{n}^{(3)}$.

Remark 3. In order to determine the tricyclic graphs which attain the minimum detour index of all graphs in $\mathscr{T}_{n}$, we just need to discuss the tricyclic graphs in $\mathscr{T}$, where $\widehat{\mathscr{T}}^{n} \in \mathscr{T}_{n}^{(3)}=$ $\left\{\mathscr{T}^{16}, \mathscr{T}^{22}, \mathscr{T}^{23}, \mathscr{T}^{24}, \mathscr{T}^{25}, \mathscr{T}^{26}\right\}$ (see Figure 13).

\section{Results and Discussion}

From the discussions of Section 2, we can verify that if $\mathscr{T} \in \mathscr{T}_{n}$ attains the minimum detour index of all graphs in $\mathscr{T}_{n}$, then $\omega(\mathscr{T})=\min \{\omega(G)\}$, where $\widehat{G} \in \mathscr{T}_{n}^{(3)}=\left\{\mathscr{T}^{16}\right.$, $\left.\mathscr{T}^{22}, \mathscr{T}^{23}, \mathscr{T}^{24}, \mathscr{T}^{25}, \mathscr{T}^{26}\right\}$.

Theorem 1. Let $\mathscr{T}_{n}^{(3)}$ be defined as in Figure 13.

(1) When $n=4$ or $n \geq 8, \mathscr{T}^{26}$ is the unique graph which attains the minimum detour index of all graphs in $\mathscr{T}_{n}$ and $\omega\left(\mathscr{T}^{26}\right)=n^{2}+4 n-14$.

(2) When $n=5$ or $n=6, \mathscr{T}^{25}$ is the unique graph which attains the minimum detour index of all graphs in $\mathscr{T}_{n}$ and $\omega\left(\mathscr{T}^{25}\right)=n^{2}+5 n-21$.

(3) When $n=7, \mathscr{T}^{25}$ and $\mathscr{T}^{26}$ are the graphs which attain the minimum detour index of all graphs in $\mathscr{T}_{n}$ and $\omega\left(\mathscr{T}^{25}\right)=\omega\left(\mathscr{T}^{26}\right)=63$.

Proof. It can be checked directly that

$$
\begin{aligned}
L\left(v_{1} \mid \mathscr{T}^{16}\right) & =n+5 \\
L\left(v_{i} \mid \mathscr{T}^{16}\right) & =3 n-1, \text { where } 2 \leq i \leq 7 \\
L\left(w \mid \mathscr{T}^{16}\right) & =2 n+3, \text { where } w \in W_{v_{1}} .
\end{aligned}
$$




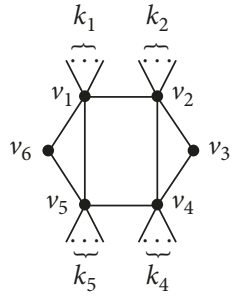

G

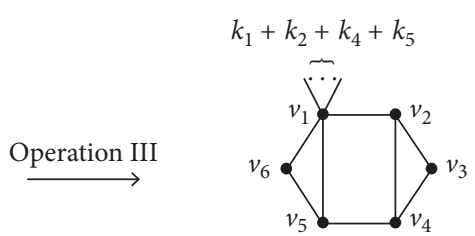

$G^{\prime}$

Figure 12: Operation IV on graph $G \in \mathscr{T}^{19}$.
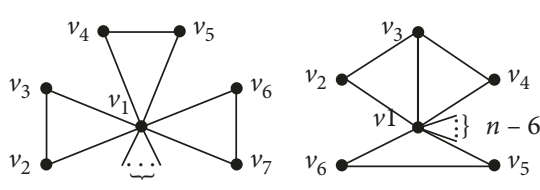

$n-7$

$\mathscr{T}^{16}(n \geq 7)$

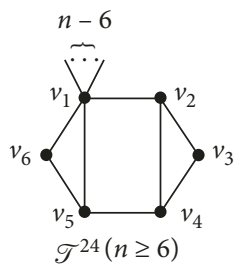

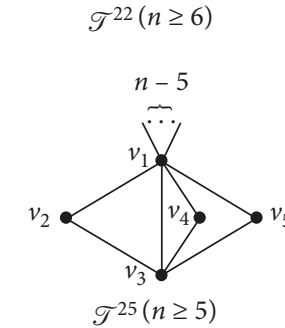

Figure 13: Graph $\mathscr{T}_{n}^{(3)}$.

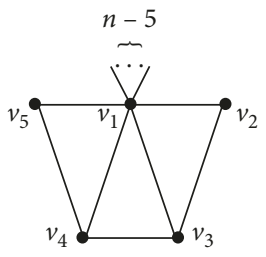

$\mathscr{T}^{23}(n \geq 5)$

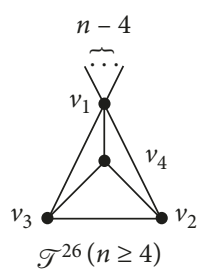

Therefore,

$$
\begin{aligned}
\omega\left(\mathscr{T}^{16}\right) & =\frac{1}{2} \sum_{u \in V\left(\mathscr{T}^{16}\right)} L\left(u \mid \mathscr{T}^{16}\right) \\
& =\frac{1}{2}[(n+5)+6(3 n-1)+(2 n+3)(n-7)] \\
& =n^{2}+4 n-11, \quad n \geq 7 .
\end{aligned}
$$

Similarly, we have

$$
\begin{aligned}
& \begin{aligned}
\omega\left(\mathscr{T}^{22}\right) & =n^{2}+5 n-15 \\
& =n^{2}+4 n+(n-15), \quad n \geq 6,
\end{aligned} \\
& \begin{aligned}
\omega\left(\mathscr{T}^{23}\right) & =n^{2}+8 n-27 \\
& =n^{2}+4 n+(4 n-27), \quad n \geq 5, \\
\omega\left(\mathscr{T}^{24}\right) & =n^{2}+15 n-57 \\
& =n^{2}+4 n+(11 n-57), \quad n \geq 6, \\
\omega\left(\mathscr{T}^{25}\right) & =n^{2}+5 n-21 \\
& =n^{2}+4 n+(n-21), \quad n \geq 5, \\
\omega\left(\mathscr{T}^{26}\right) & =n^{2}+4 n-14, \quad n \geq 4 .
\end{aligned}
\end{aligned}
$$

(1) When $n=4$ or $n \geq 8$, obviously, $\mathscr{T}^{26}$ is the unique graph which attains the minimum detour index of all graphs in $\mathscr{T}_{n}$ and $\omega\left(\mathscr{T}^{26}\right)=n^{2}+4 n-14$.

(2) When $n=5$ or $n=6$, obviously, $\mathscr{T}^{25}$ is the unique graph which attains the minimum detour index of all graphs in $\mathscr{T}_{n}$ and $\omega\left(\mathscr{T}^{25}\right)=n^{2}+5 n-21$.

(3) When $n=7$, obviously, $\mathscr{T}^{25}$ and $\mathscr{T}^{26}$ are the graphs which attain the minimum detour index of all graphs in $\mathscr{T}_{n}$ and $\omega\left(\mathscr{T}^{25}\right)=\omega\left(\mathscr{T}^{26}\right)=63$.

\section{Conclusions}

Mathematical chemistry is an area of research in chemistry in which mathematical tools are used to solve problems of chemistry. Chemical graph theory is an important area of research in mathematical chemistry which deals with topology of molecular structures such as the mathematical study of isomerism and the development of topological descriptors or indices. In this paper, we first introduce some useful graph transformations, and then we determine the minimum detour index of all tricyclic graphs. In addition, all the corresponding extremal graphs are characterized. 


\section{Data Availability}

The data used to support the findings of this study are available from the corresponding author upon request.

\section{Conflicts of Interest}

The authors declare no conflicts of interest regarding the content and implications of this manuscript.

\section{Acknowledgments}

This work was supported by the open project of Anhui University (no. KF2019A01), the Natural Science Research Foundation of the Department of Education of Anhui Province (no. KJ2019A0817), and the Project of Teaching Team of Chizhou University (no. 2016XJXTD02).

\section{References}

[1] W. Fang, H. Yu, Y. Gao, G. Jing, and Z. Li, "Maximum Balaban index and sum-Balaban index of tricyclic graphs," MATCH Communications in Mathematical and in Computer Chemistry, vol. 79, pp. 717-742, 2018.

[2] D. Wang, S. Tan, and L. Zhu, "On the lower and upper bounds for different indices of tricyclic graphs," Journal of Applied Mathematics and Computing, vol. 51, no. 1-2, pp. 1-11, 2016.

[3] F. Buckley and F. Harary, Distance in Graphs, AddisonWesley, Reading, MA, USA, 1990.

[4] O. Ivanciuc and A. T. Balaban, "Design of topological indices-part 8: path matrices and derived molecular graph invariants," MATCH Communications in Mathematical and in Computer Chemistry, vol. 30, pp. 141-152, 1994.

[5] G. Rücker and C. Rücker, "Symmetry-aided computation of the detour matrix and the detour index," Journal of Chemical Information and Computer Sciences, vol. 38, no. 4, pp. 710714, 1998.

[6] I. Lukovits, "The detour index," Croatica Chemica Acta, vol. 69, pp. 873-882, 1996.

[7] J.-B. Liu, M. Younas, M. Habib, M. Yousaf, and W. Nazeer, "M-Polynomials and degree-based topological indices of $\mathrm{VC}_{5} \mathrm{C}_{7}[\mathrm{p}, \mathrm{q}]$ and $\mathrm{HC}_{5} \mathrm{C}_{7}[\mathrm{p}, \mathrm{q}]$ nanotubes," IEEE Access, vol. 7, pp. 41125-41132, 2019.

[8] X. Qi and B. Zhou, "Minimum detour index of unicyclic graphs with given maximum degree," Ars Combinatoria, vol. 102, pp. 193-200, 2011.

[9] X. Qi, "Detour index of bicyclic graphs," Utilitas Mathematica, vol. 90, pp. 101-113, 2013.

[10] N. Trinajstić, S. Nikolić, B. Lǔcić, D. Amić, and Z. Mihalić, "The detour matrix in chemistry," Journal of Chemical Information and Computer Sciences, vol. 37, no. 4, pp. 631-638, 1997.

[11] J.-B. Liu, C. Wang, and S. Wang, "Zagreb indices and multiplicative zagreb indices of eulerian graphs," Bulletin of the Malaysian Mathematical Sciences Society, vol. 42, pp. 67-78, 2019.

[12] J.-B. Liu and X. F. Pan, "Minimizing Kirchhoffindex among graphs with a given vertex bipartiteness," Applied Mathematics and Computation, vol. 291, pp. 84-88, 2016.

[13] J.-B. Liu, J. Zhao, J. Min, and J. Cao, "On the Hosoya index of graphs formed by a fractal graph," Fractals: Complex Geometry, Patterns, and Scaling in Nature and Society, 2019.
[14] H. Deng, "On the Balaban index of trees," MATCH Communications in Mathematical and in Computer Chemistry, vol. 66, pp. 253-260, 2011.

[15] H. Deng, "On the sum-Balaban index," MATCH Communications in Mathematical and in Computer Chemistry, vol. 66, pp. 273-284, 2011.

[16] W. Fang, H. Yu, Y. Gao, G. Jing, Z. Li, and X. Li, "Minimum Detour index of cactus graphs," Ars Combinatoria, vol. 144, pp. 293-307, 2019. 

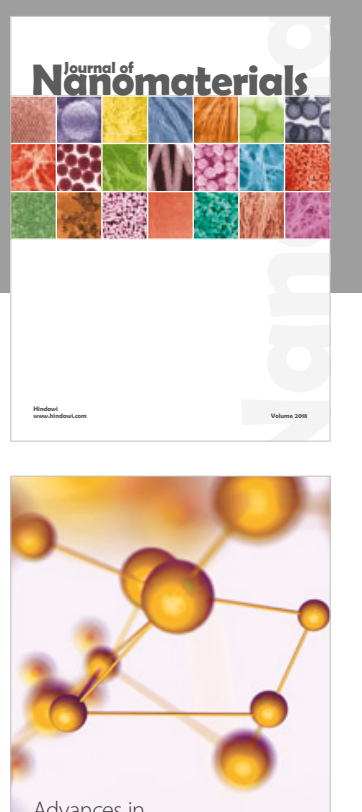

Physical Chemistry
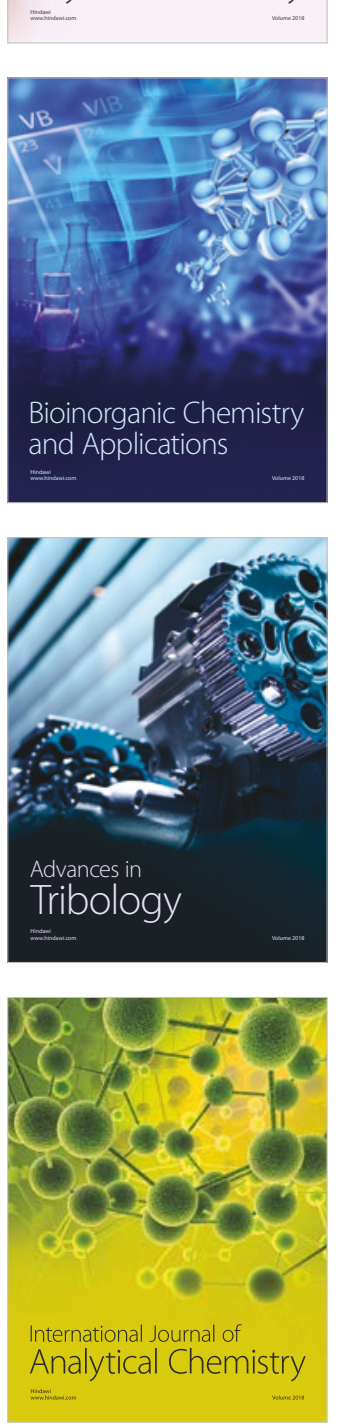

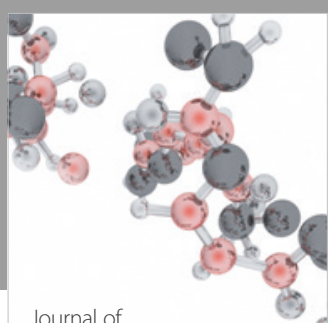

Analytical Methods

in Chemistry

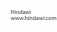

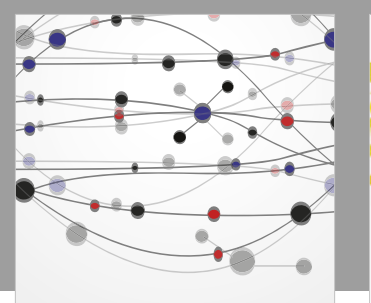

The Scientific World Journal

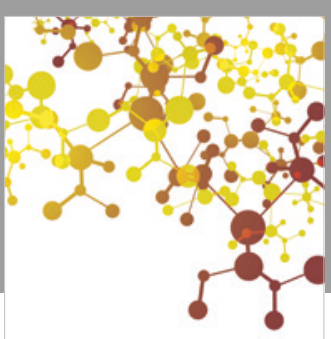

Journal of

Applied Chemistry
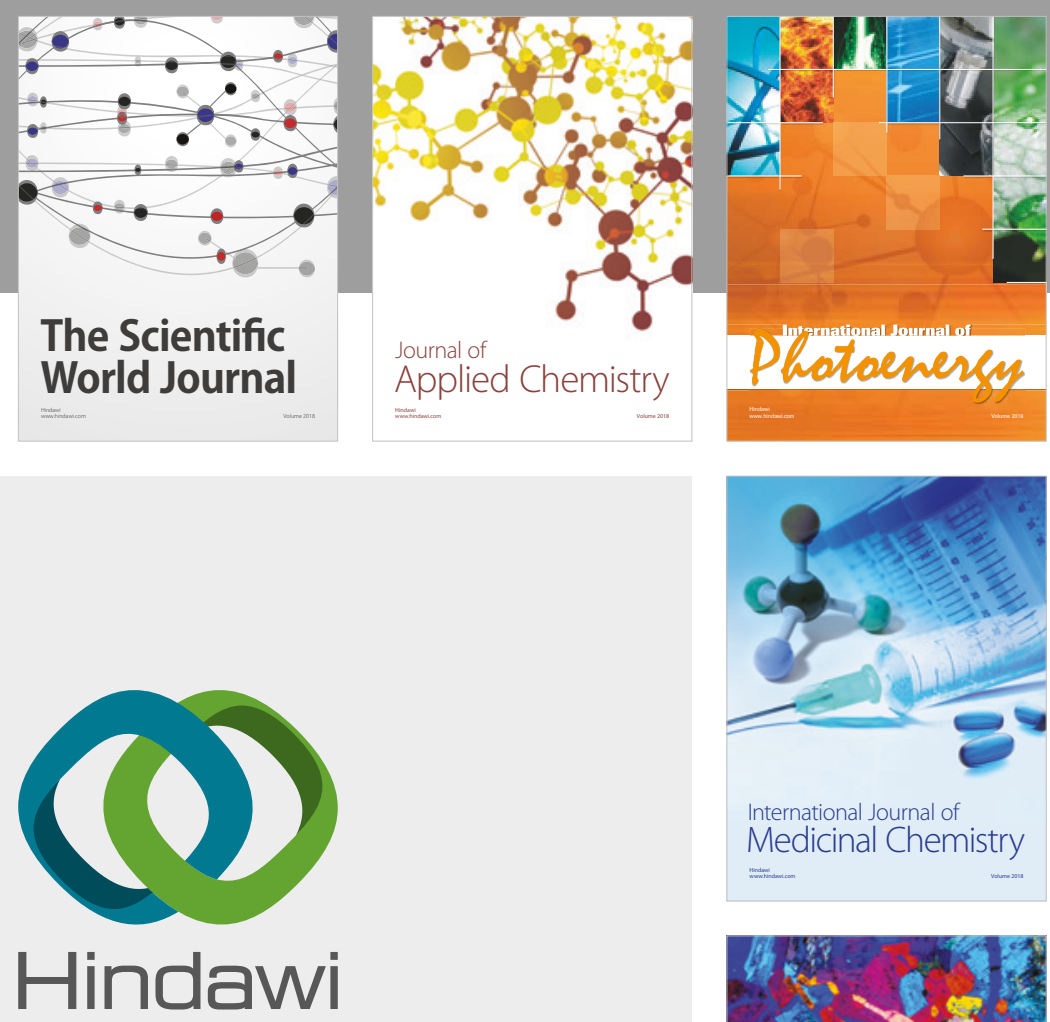

Submit your manuscripts at

www.hindawi.com
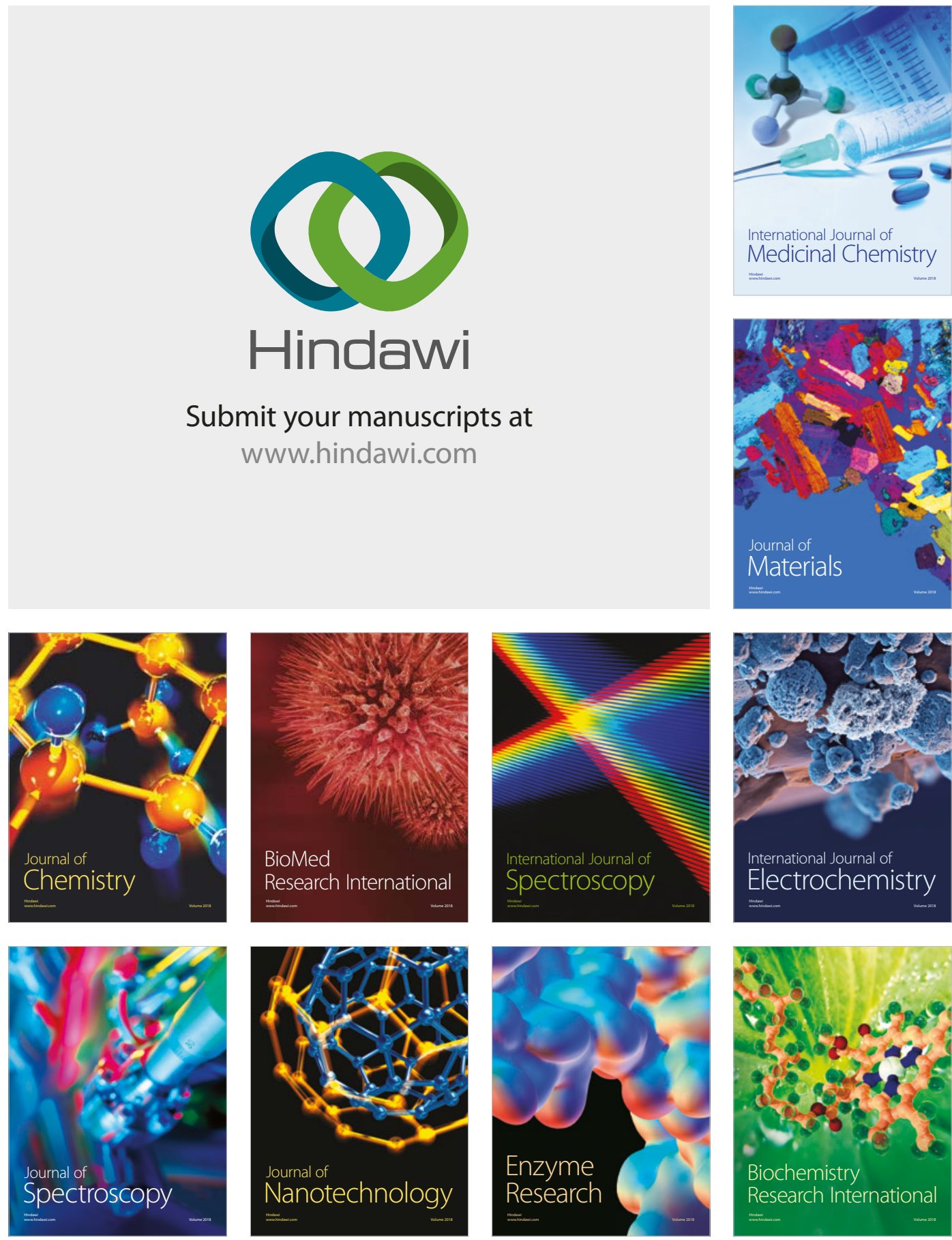
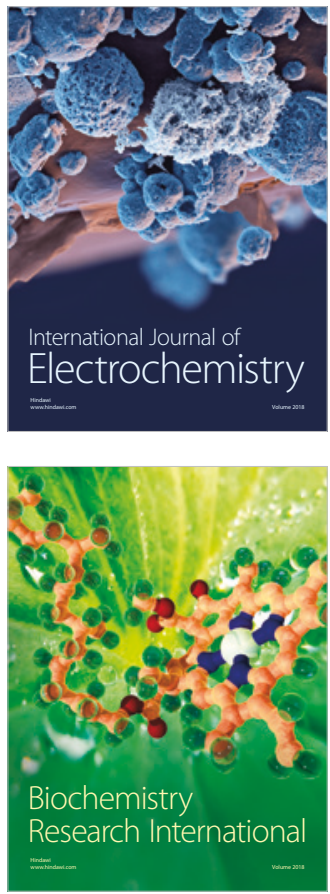